\title{
Phase transitions in the interacting boson fermion model: The $\gamma$-unstable case
}

\author{
C. E. Alonso, ${ }^{1}$ J. M. Arias, ${ }^{1}$ L. Fortunato,${ }^{2}$ and A. Vitturi ${ }^{2}$ \\ ${ }^{1}$ Departamento de Física Atómica, Molecular y Nuclear, Facultad de Física, Universidad de Sevilla, Apartado 1065, E-41080 Sevilla, Spain \\ ${ }^{2}$ Dipartimento di Fisica Galileo Galilei and INFN, Via Marzolo 8, I-35131 Padova, Italy
}

(Received 5 September 2005; published 27 December 2005)

\begin{abstract}
The phase transition around the critical point in the evolution from spherical to deformed $\gamma$-unstable shapes is investigated in odd nuclei within the interacting boson fermion model. We consider the particular case of an odd $j=3 / 2$ particle coupled to an even-even boson core that undergoes a transition from spherical U(5) to $\gamma$-unstable $\mathrm{O}(6)$ situation. The particular choice of the $j=3 / 2$ orbital preserves in the odd case the condition of $\gamma$-instability of the system. As a consequence, energy spectrum and electromagnetic transitions, in correspondence of the critical point, display behaviors qualitatively similar to those of the even core. The results are also in qualitative agreement with the recently proposed E(5/4) model, although few differences are present, due to the different nature of the two schemes.
\end{abstract}

DOI: 10.1103/PhysRevC.72.061302

PACS number(s): 21.60.Fw, 21.60.Ev

The study of phase transitions has recently received particular attention in nuclear structure. The concept of critical point symmetry has been first proposed in a number of cases by Iachello [1-3]. These symmetries apply when a quantal system undergoes transitions between traditional dynamical symmetries, as for example those characterizing situations described in terms of harmonic vibrations or rigid rotations. Although these symmetries have been obtained within the formalism based on the Bohr Hamiltonian [4], their concept has also been used in connection with the interacting boson model (IBM) [5].

One of these critical point symmetries is associated with the transition between spherical and $\gamma$-unstable shapes. Within the IBM this can be obtained, for example, from the Hamiltonian

$$
H_{B}=x \hat{n}_{d}-\frac{1-x}{N} \hat{Q}_{B} \cdot \hat{Q}_{B},
$$

which produces, varying the parameter $x$ from 1 to 0 , a transition between the two extreme situations characteristic of $\mathrm{U}(5)$ and $\mathrm{O}(6)$ symmetries. The corresponding second-order shape phase transition has been investigated within the Bohr collective model in Ref. [6]. The operators appearing in the Hamiltonian above are given by

$$
\begin{aligned}
\hat{n}_{d} & =\sum_{\mu} d_{\mu}^{\dagger} d_{\mu}, \\
\hat{Q}_{B} & =\left(s^{\dagger} \times \tilde{d}+d^{\dagger} \times \tilde{s}\right)^{(2)},
\end{aligned}
$$

and $N$ is the total number of bosons. For any value of $x$ this Hamiltonian maintains the typical degeneracies of the $\mathrm{O}(5)$ symmetry. Consistently with this, within the IBM coherent state formalism [7-9], this Hamiltonian always produces an energy surface which is independent of the $\gamma$ degree of freedom. In the $\beta$ variable, the energy surface displays a spherical minimum in $\beta=0$ for $x$ larger than the critical value $x_{c}=\frac{4 N-8}{5 N-8}$, while having a deformed minimum for values of $x$ smaller than the critical value. At the critical point, the energy surface acquires a $\beta^{4}$ behavior $[10,11]$, which is approximated by an infinite square well in the $\mathrm{E}(5)$ critical point symmetry [1] within the framework of the collective Bohr Hamiltonian.
Recently Iachello has discussed a supersymmetrical extension of this concept, introducing the so-called E(5/4) model, where the boson part has a $\gamma$-independent square well potential and the boson-fermion coupling is taken as a spin (5) scalar interaction [12]. This solution describes the spectral properties of odd-even nuclei at the transition between spherical and $\gamma$-unstable shapes. In this Rapid Communication we want to discuss the evolution of odd nuclei and the corresponding behavior at the critical point, within the framework of the interacting boson fermion model (IBFM) [13], in which a single fermion is coupled to the even-even bosonic core. The system will then be described by the Hamiltonian

$$
H=H_{B}+H_{F}+V_{B F},
$$

where the term $V_{B F}$ couples the bosonic and fermionic parts. In our case we will assume the boson Hamiltonian to be of the form given in Eq. (1). For the fermion and boson-fermion parts we will take the particular choice of a particle moving in a single $j$-shell $j=3 / 2$ and a coupling term of the form

$$
V_{B F}=-2 \frac{1-x}{N} \hat{Q}_{B} \cdot \hat{q}_{F},
$$

where $\hat{Q}_{B}$ [taken of the form given in Eq. (3)] and $\hat{q}_{F}=$ $\left(a_{3 / 2}^{\dagger} \times \tilde{a}_{3 / 2}\right)^{(2)}$ are the boson and fermion quadrupole operators, respectively.

This choice of the fermion space and the boson-fermion interaction is such that one recovers, in the extreme cases, the Bose-Fermi symmetry [13] associated with the $\operatorname{spin}^{B F(6)}$ group (for $x=0$ ) and the vibrational $U^{B}(5) \otimes S U^{F}$ (4) case (for $x=1)$. In analogy with the overall $\mathrm{O}(5)$ structure in the even case, this selection guarantees the preservation of the degeneracies associated with the $\operatorname{spin}^{B F}(5)$ symmetry for any value of $x$. This can be more clearly seen within the intrinsic frame formalism [7-9]. In order to get energy eigenvalues for the case of a $j=3 / 2$ particle coupled to the ground state $\left|\Phi_{B}(\beta, \gamma)\right\rangle$ boson condensate one has to diagonalize the Hamiltonian in a space of dimension four with basis vectors: $\left|\Phi_{B}(\beta, \gamma)\right\rangle \otimes|j, m\rangle$. The pure boson part for the case considered here is only a function of $\beta$ and gives a 

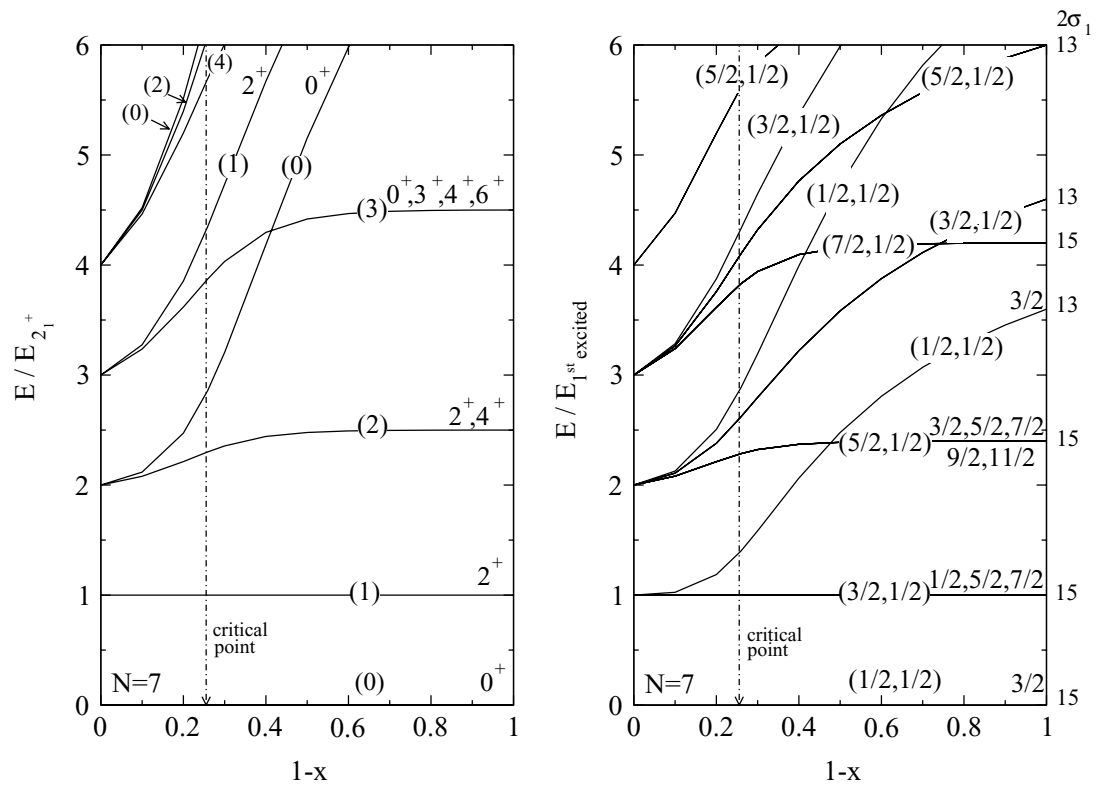

FIG. 1. Energy levels (normalized to the energy of the first excited state) for the even and odd systems are displayed as a function of the parameter $(1-x)$ for the boson (1) and boson-fermion (4) Hamiltonians. A number $N=7$ of bosons has been assumed in both cases, while the odd particle has been taken in the $j=3 / 2$ orbital. In the left panel (even case) we indicate for each level the $\tau$ quantum number (in parenthesis), spin and parity. In the right panel (odd case) we quote the $\left(\tau_{1}, \tau_{2}\right)$ quantum numbers (in parenthesis) and spin. In the extreme $x=0$ case we also indicate the $\sigma_{1}$ quantum number. The position of the even critical point is marked. global diagonal contribution. The pure fermionic part is just a constant. Thus, the only remaining part, $V_{B F}$, has to be diagonalized. Its eigenvalues, doubly degenerate, turn out to be $\gamma$-independent,

$$
E_{ \pm}(\beta, \gamma)=E_{ \pm}(\beta)= \pm 2 \frac{(1-x) \beta}{1+\beta^{2}}
$$

In other words, the addition of the odd particle does not destroy the $\gamma$-instability of the system, giving rise to energy surfaces for the different odd intrinsic states that are still $\gamma$-independent [14]. The particular behavior of the $j=3 / 2$ orbital was first put in evidence by Bayman and Silverberg [15] within the collective model.

The resulting energy spectra in the odd system are shown in the right panel of Fig. 1 as a function of the control parameter $1-x$. The total number of bosons, $N$, has been assumed to be equal to 7 . For a better comparison, we also show in the left panel of the figure the corresponding evolution of the spectrum in the even core. It should be remembered that the use of a finite number of bosons does not lead to the same results as the corresponding ones obtained in the equivalent situation within the Bohr Hamiltonian, which is only reached in the limit of infinite number of bosons. Note also that the geometrical limit obtained from the boson Hamiltonian (1) for large values of $N$ corresponds to the case of the collective potential behaving as $\beta^{4}$, and not precisely to the $\mathrm{E}(5)$ case, that corresponds to the infinite square well. The level evolution in the odd case shows a behavior qualitatively similar to that of the even case. The group structure of $\operatorname{spin}^{B F(5)}$ with respect to $\mathrm{O}(5)$ simply leads to a richer pattern for the fermion case and slightly different ratios for the energy levels. For example in the limiting $x$ $=0$ case, corresponding to $\operatorname{spin}^{B F(6)}$ and $\mathrm{O}(6)$ symmetry groups for the odd and even nuclei, respectively, we obtain for the "ground bands," with maximum $\sigma$ values $\left(\sigma_{1}=N+1 / 2\right.$ and $\sigma=N$ for odd and even nuclei, respectively), the ratios $E\left(\tau_{1}=5 / 2, \tau_{2}=1 / 2\right) / E\left(\tau_{1}=3 / 2, \tau_{2}=1 / 2\right)=2.4$ and
$E\left(\tau_{1}=7 / 2, \tau_{2}=1 / 2\right) / E\left(\tau_{1}=3 / 2, \tau_{2}=1 / 2\right)=4.2$ with respect to the values $E(\tau=2) / E(\tau=1)=2.5$ and $E(\tau=3) / E(\tau=1)=4.5$ of the even case.

As in the even case, the levels that in the extreme $x=0$ limit eventually correspond to the "ground band" $\left(\sigma_{1}=N+1 / 2\right)$ show a rather smooth (or even flat) behavior, aside from the changes around the critical point. Instead, the levels that will end up with other values of $\sigma_{1}$ (as the levels corresponding to smaller values of $\sigma$ in the even case) show a more violent variation in the whole range. A typical case is the second $\left(\tau_{1}=1 / 2, \tau_{2}=1 / 2\right) j=3 / 2$ state that starts at one-phonon energy in the vibrational limit to end up as the third $j=$ $3 / 2$ state in the opposite limit. The position of this $3 / 2$ state is the key element to characterize the particular situation and its position along the transitional path. The position of this state plays the same role as the key position of the first excited $0^{+}$state in even nuclei. The smoother behavior of the ground band levels with respect to the other bands confirms the fact that to establish a definite critical situation it is not at all sufficient to rely just on the sequence of energy levels in the "ground" band, and that some of the claims for the occurrence of definite transitional symmetries have to be taken with serious caution.

Crucial and selective information on nuclear spectra comes from the transition probabilities, in particular from the electric quadrupole ones. These are obtained in terms of the matrix elements of the electric quadrupole operator. In our IBFM case this operator is given by

$$
\hat{Q}_{B F}=\hat{Q}_{B}+\hat{q}_{F}=\left(s^{\dagger} \times \tilde{d}+d^{\dagger} \times \tilde{s}\right)^{(2)}+\left(a_{j}^{\dagger} \times \tilde{a}_{j}\right)^{(2)} .
$$

Note that, consistently with the boson quadrupole operator used in the Hamiltonian, we have not included any $\left(d^{\dagger} \times \tilde{d}\right)^{(2)}$ term in the transition operator.

Values of the individual transition probabilities, state by state, for the odd nucleus at the critical point situation are shown in Fig. 2. In it, we have plotted the lowest six multiplets, which have been arbitrarily split in order to show the possible 


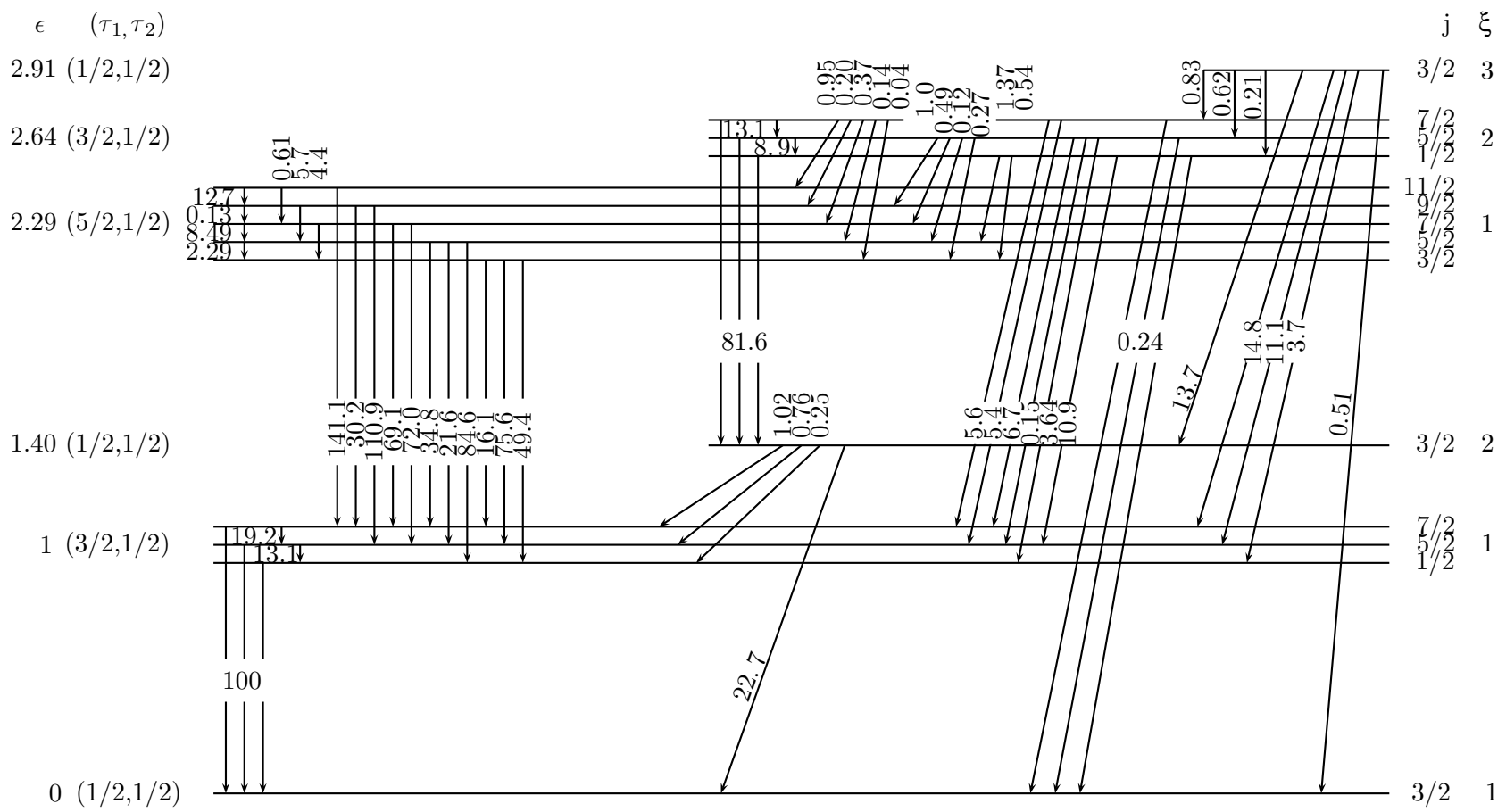

FIG. 2. Energy levels and quadrupole transition rates $B(E 2 \downarrow)$ for the odd system at the core critical point. For illustration purposes the various multiplets, labeled by the $\left(\tau_{1}, \tau_{2}\right)$ quantum numbers in parenthesis, have been arbitrarily split according to their $j$ quantum number. The label on the extreme left is the energy in relative units, while the label at the extreme right corresponds to the label $\xi$, used in Ref. [12]. $B(E 2 \downarrow)$ 's have been normalized to the value 100 for the transitions (with equal strengths) between the states of the first multiplet and the ground state.

E2 transitions between the different states. In the figure the levels have different lengths depending on the $\xi$ value, which labels diferent families and it is shown along with the spin of the state (we conform here to the notation introduced in Ref. [12]). Although the general pattern remains the same, the detailed energy sequence depends on the number of interacting bosons, $N$. Owing to the properties of the $\operatorname{spin}^{B F(5)}$ group, the $\Delta \tau_{1}=0, \pm 1, \Delta \tau_{2}=0$ selection rule still hold. It can be observed that $E 2$ transitions are stronger between states with the same $\xi$ value and $\Delta \tau_{1}=1$. Transitions between states pertaining to families with different $\xi$ are one or two orders of magnitude smaller. Transitions between states with the same $\xi$ and $\tau_{1}$ values, but different spins, corresponding to the same multiplets are also one or two orders of magnitude smaller than the ones between different multiplets in the same band.

The allowed quadrupole transitions are sketched in Fig. 3 for the odd system for the $\operatorname{spin}^{B F(6)}$ case and for the critical point situation, and for the even case at the critical point. In addition to the difference in the level sequence, in the second case transitions are allowed between different bands (although weaker than inband transitions, as already mentioned), in a similar way as interband transitions are allowed at the critical point for the even case (as for example between the second $0^{+}$, with $\tau=0$, and the first $2^{+}$, with $\tau=1$, as shown in the figure).

The comparison of our critical point solution with Iachello's one evidences a similar overall organization of the spectrum, although with sizable differences in the relative position of the different $\xi$ bands. It must be recalled that, in principle, we should compare the $\mathrm{E}(5 / 4)$ solution with the large $N$ limit of the interacting boson model, while we have concentrated, along this paper, on the $N=7$ case. The first $\tau_{1}=5 / 2$ multiplet in the $\mathrm{E}(5 / 4)$ lies at around 2.20 , close to the value 2.29 obtained in our model. On the other hand, the two first multiplets of the $\xi=2$ family of excited states of the $E(5 / 4)$ solution lie at around 3.33 and 5.02, compared with the corresponding values 1.40 and 2.64 of our model. This inversion of the position of

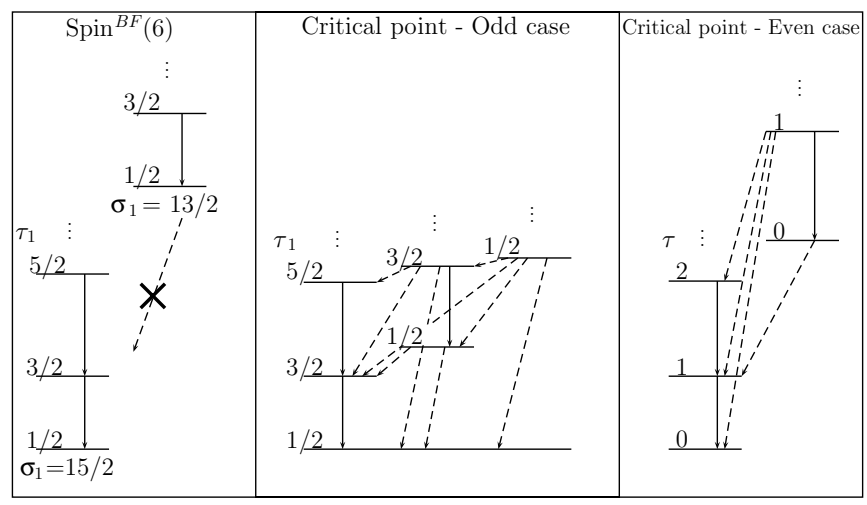

FIG. 3. Schematic illustration of allowed quadrupole transitions for the odd system at the $\operatorname{spin}^{B F}(6)$ (left panel) and critical point (middle panel) cases, and for the even case at the critical point (right panel). Solid lines indicate stronger in-band transitions with respect to the interband transitions shown as dashed lines. Horizontal lines correspond to multiplets including several $j$ values. 

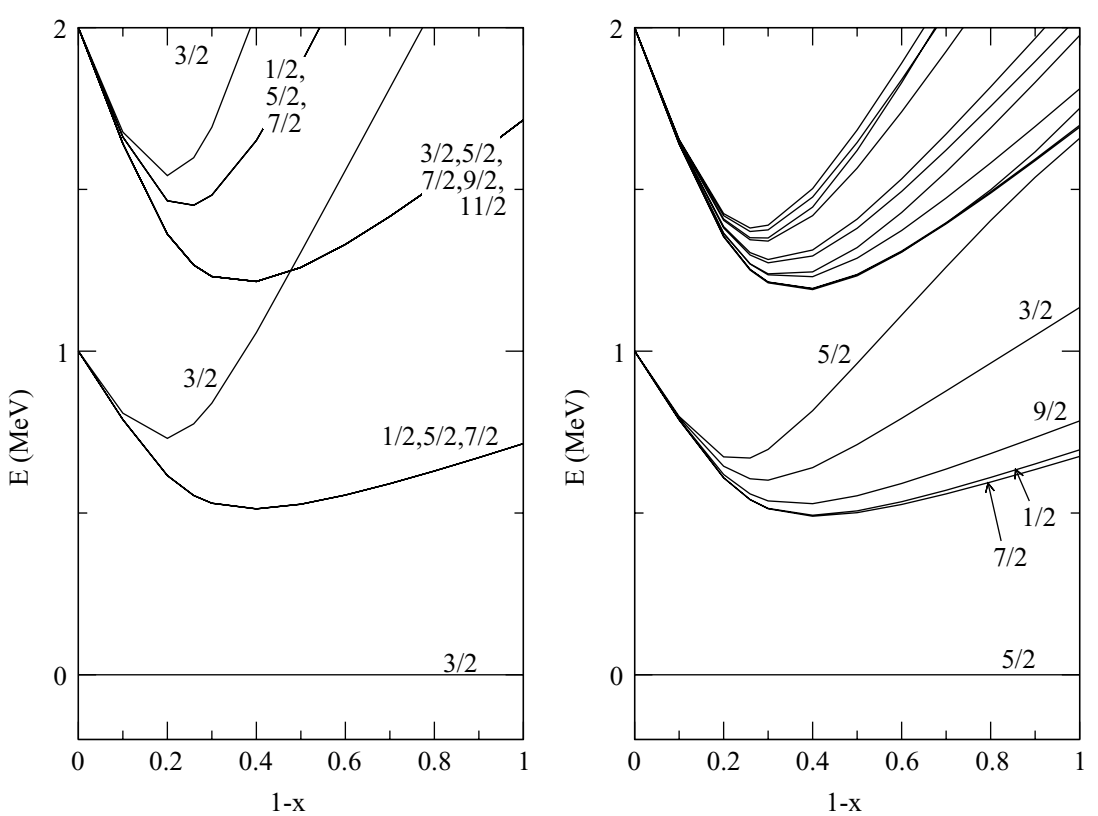

FIG. 4. Energy levels for the odd system are displayed as a function of the parameter $(1-x)$ for the boson-fermion Hamiltonian (4). A number $N=7$ of bosons has been assumed, while the odd particle has been taken in the $j=$ $3 / 2$ orbital (left panel) and $j=5 / 2$ orbital (right panel). The spin quantum number is indicated for each state. the $\xi=1, \tau_{1}=5 / 2$ and $\xi=2, \tau_{2}=1 / 2$ multiplets is the first evident difference between the two models. Another important difference is seen in the relative $B(E 2)$ values: although in-band transitions display comparable values, significant discrepancies are observed in interband transition (notably the transitions between the $\xi=2, \tau_{1}=1 / 2$ state and the two lowest multiplets of the ground state band).

Preserving the degeneracies associated with the $\operatorname{spin}^{B F(5)}$ symmetry is not an exclusive property of the $j=3 / 2$ orbitals. Other cases are known in the literature, for example the case of the odd particle moving in the $j=1 / 2,3 / 2,5 / 2$ orbitals, under the condition of special values for the fermion quadrupole matrix elements. Also in this case one recovers, for the $x=0$ limiting case, the Bose-Fermi symmetry behavior. But this does not happen in more general cases with other combinations of orbitals or fermion quadrupole matrix elements [16]. To give an idea, we present in Fig. 4 the evolution of the spectrum as a function of the control parameter $1-x$ in the cases of a single $j=3 / 2$ and $j=5 / 2$. The figure shows a spectrum for $j=5 / 2$ (right panel) that is qualitatively similar to the one displayed in the left panel for $j=3 / 2$, but is more complex due to the removal of all degeneracies.
To summarize, we have considered, within the interacting boson fermion model, the coupling of an odd $j=3 / 2$ particle to a boson core that undergoes a transition from spherical $\mathrm{U}(5)$ to $\gamma$-unstable $\mathrm{O}(6)$ character. The particular choice of the Hamiltonian and of the $j=3 / 2$ orbital preserves in the odd case the condition of $\gamma$-instability of the system, and it is reflected in the preservation of the degeneracies associated with the $\operatorname{spin}^{B F(5)}$ symmetry. As a consequence, the energy spectrum and the electromagnetic transitions for the odd nucleus with a critical core display behaviors qualitatively similar to those characterizing the phase transition in the even core. We have compared our results with the recently proposed E(5/4) approach, based on the Bohr Hamiltonian. Both approaches display similar qualitative pictures, although we evidence a number of quantitative differences, that can be traced back to the different nature of the two schemes.

We acknowledge enlightening discussions with J. Barea and B. F. Bayman. This work was supported by the Italian-Spanish agreement INFN-CICYT and by the Spanish DGICYT under project number FIS2005-01105.
[1] F. Iachello, Phys. Rev. Lett. 85, 3580 (2000).

[2] F. Iachello, Phys. Rev. Lett. 87, 052502 (2001).

[3] F. Iachello, Phys. Rev. Lett. 91, 132502 (2003).

[4] A. Bohr and B. Mottelson, Nuclear Structure: Vol II, Nuclear Deformations (Benjamin, Reading, MA, 1975).

[5] F. Iachello and A. Arima, The Interacting Boson-Model (Cambridge University Press, Cambridge, 1987).

[6] P. S. Turner and D. J. Rowe, Nucl. Phys. A756, 333 (2005).

[7] J. N. Ginocchio and M. W. Kirson, Nucl. Phys. A350, 31 (1980).

[8] A. E. L. Dieperink, O. Scholten, and F. Iachello, Phys. Rev. Lett. 44, 1747 (1980).

[9] A. Bohr and B. Mottelson, Phys. Scr. 22, 468 (1980).
[10] J. M. Arias, C. E. Alonso, A. Vitturi, J. E. García-Ramos, J. Dukelsky, and A. Frank, Phys. Rev. C 68, 041302(R) (2003).

[11] J. E. Garcia-Ramos, J. Dukelsky, and J. M. Arias, Phys. Rev. C 72, 037301 (2005).

[12] F. Iachello, Phys. Rev. Lett. 95, 052503 (2005).

[13] F. Iachello and P. Van Isacker, The Interacting Boson Fermion Model (Cambridge University Press, Cambridge, 1991).

[14] C. E. Alonso, J. M. Arias, F. Iachello, and A. Vitturi, Nucl. Phys. A539, 59 (1992).

[15] B. F. Bayman and L. Silverberg, Nucl. Phys. 16, 625 (1960).

[16] J. Jolie, S. Heinze, P. Van Isacker, and R. F. Casten, Phys. Rev. C 70, 011305(R) (2004). 